\title{
Circularly polarized emission from the transient bursting radio source GCRT J1745-3009
}

\author{
Subhashis Roy \\ NCRA-TIFR, Pune-411007, India \\ roy@ncra.tifr.res.in \\ Scott D. Hyman \\ Department of Physics and Engineering, Sweet Briar College, Sweet Briar, VA 24595 \\ shyman@sbc.edu \\ Sabyasachi Pal \\ International Centre for Radio Astronomy Research, University of Western Australia, 35 \\ Stirling Highway, Crawley, 6009, Australia \\ T. Joseph W. Lazio \\ Remote Sensing Division, Naval Research Laboratory, Washington, DC 20375-5351 \\ joseph.lazio@nrl.navy.mil \\ Paul S. Ray \\ Space Science Division, Naval Research Laboratory, Washington, DC 20375-5352 \\ Paul.Ray@nrl.navy.mil \\ Namir E. Kassim \\ Remote Sensing Division, Naval Research Laboratory, Washington, DC 20375-5351 \\ namir.kassim@nrl.navy .mil
}

Received _ ; accepted _ 


\begin{abstract}
We report detection of strong circularly polarized emission from the transient bursting source GCRT J1745-3009 based on new analysis of $325 \mathrm{MHz}$ GMRT observations conducted on 28 September 2003. We place $8 \mathrm{R}_{\odot}$ as the upper limit on the size of the emission region. The implied high brightness temperature required for an object beyond $1 \mathrm{pc}$ and the high fraction of circular polarization firmly establish the emission as coherent. Electron cyclotron or plasma emission from a highly subsolar magnetically dominated dwarf located $\leq 4 \mathrm{kpc}$ away could have given rise to the GCRT radio emission.
\end{abstract}

Subject headings: Galaxy: center - radio continuum: general - stars: individual (GCRT J1745-3009) - stars: variables: other 


\section{Introduction:}

The present generation of largely parabolic dish-based radio interferometers and data analysis procedures are poorly optimized for discovering transient emission. Consequently, the characteristics of transient radio source populations remain highly undersampled. Nevertheless, dedicated programs are revealing novel radio transients with unique emission characteristics, including GCRT J1745-3009. Discovered in $325 \mathrm{MHz}$ archival VLA data taken in September 2002, this source exhibited 10 minute long, 1 Jy peaked bursts with a $\sim 77$ minute period over the $\sim 7$ hour baseline available in the discovery data (Hyman et al. 2005). The measured time scales of the bursts implied a brightness temperature exceeding the $10^{12} \mathrm{~K}$ Compton limit for a distance beyond $\sim 100$ pc. As discussed in Hyman et al. (2005), its characteristics did not match any known mechanisms of emission in transient compact sources. As a result, GCRT J1745-3009 appeared to represent a member of a new class of coherently emitting objects.

Search to detect additional bursts from the source in archival data sets were made, resulting in its re-detection for $\sim 2$ minutes at $325 \mathrm{MHz}$ Giant metrewave radio telescope (GMRT) observation in September 2003 (Hyman et al. 2006) and in March 2004 (Hyman et al. 2007). At the last known epoch of emission detected in 2004, the source exhibited an unusually steep spectrum with alpha $=-13 \pm 3\left(\mathrm{~S}(\nu) \propto \nu^{\alpha}\right)($ Hyman et al. 2007).

Several theories have been proposed to explain the emission from GCRT J1745-3009 (see Hvman et al. (2007) and references therein). These include nulling (Kulkarni \& Phinney 2005), double (Turolla et al. 2005), precessing (Zhu \& Xu 2006) and a transient white

dwarf pulsar (Zhang \& Gil 2005). Also, Hallinan et al. (2007) suggested a nearby ultracool dwarf as its progenitor. Unfortunately, non-detection of GCRT J1745-3009 at frequencies other than $325 \mathrm{MHz}$ (Kaplan et al. 2008) hindered attempts to discover its progenitor. Its 
brightness temperature in possible excess of the Compton limit suggests coherent processes such as electron cyclotron maser emission or plasma emission. Notably, both of these mechanisms produce circularly polarized emission, motivating us to re-examine earlier detections of GCRT J1745-3009 in search of its signature. We have reanalyzed the 325 MHz GMRT data from September 2003, the detection with the highest signal to noise ratio per integration time available. The subsequent sections are arranged as follows - in Sect. 2 and 3 we describe our reanalysis procedure and its results, respectively. The interpretation of these results are presented in Sect. 4 and conclusions in Sect. 5.

\section{Observations and data analysis:}

During the observations, the pointing center of the GMRT antennas was $0.5^{\circ}$ West of GCRT J1745-3009 (hereafter GCRT). The unpolarized calibrator 3C48 was observed for

absolute gain calibration - see Hyman et al. 2006 for further details of the observation and total intensity calibration. At the time of these observations, the full polarization mode of the GMRT was unavailable and only parallel-hand correlations (Stokes RR and LL) were recorded. If the polarization leakage coefficients of antenna ' $\mathrm{i}$ ' is denoted by $\mathrm{D}_{i}$, then the observed right circularly polarized signal on the $i$ - $j$ baseline is

$R_{i}^{\prime} \cdot R_{j}^{\prime *}=\left(1-D_{i}-D_{j}^{*}\right) \cdot R_{i} \cdot R_{j}^{*}+R_{i} \cdot\left(D_{j} L_{j}\right)^{*}+\left(D_{i} \cdot L_{i}\right) \cdot R_{j}^{*}+\left(D_{i} R_{i}\right) \cdot\left(D_{j} \cdot R_{j}\right)^{*}+\left(D_{i} \cdot L_{i}\right) \cdot\left(D_{j} \cdot L_{j}\right)^{*}$

In general, the quantities are complex; we denote the 'true' values without primes and the measured quantities with primes; and a similar equation holds for the left circularly polarized signal $L_{i}^{\prime} L_{j}^{\prime *}$. During standard calibration without full polarization information, the leakage terms are considered small and discarded. However, the above equation shows that even for an unpolarized source the last two terms $\left[\left(D_{i} R_{i}\right) \cdot\left(D_{j} R_{j}\right)^{*}+\left(D_{i} L_{i}\right) \cdot\left(D_{j} L_{j}\right)^{*}\right]$ lead to amplitude closure errors. If the source is linearly polarized $\left(R_{i} \cdot L_{j}^{*} \neq 0\right)$, additional 
errors are added.

To determine magnitudes of these leakage coefficients, the connections for the 2 polarizations of a single antenna is reversed. This allows acquiring cross products of opposite handed polarizations of one antenna with the rest of the antennas. Observations of strong unpolarized calibrators have shown that $D_{i} \lesssim 0.1$ at $325 \mathrm{MHz}$. Since the leakage terms for different antennas are expected to be uncorrelated, the estimated error in Stokes $\mathrm{V}$ in the image plane for even a $100 \%$ linearly polarized source will be $<5 \%$ of Stokes $\mathrm{I}$. Moreover, the polarization leakage in antennas cannot add coherently in this case, indicating absence of any significant spurious small diameter source in Stokes V in the image plane. However, the above analysis is valid for a source along the axis of the antennas and the GCRT was off-axis by $0.5^{\circ}$. Antenna off-axis positions may suffer from correlated patterns among the antennas (e.g., different FWHM of the right and left circularly polarized primary beams) leading to non Zero Stokes V. Therefore, we obtained supplementary polarization calibration observations of the unpolarized calibrator 3C48 at $325 \mathrm{MHz}$ with 20 different angular offsets within the FWHM of the antenna primary beam. Fig. 1 shows that the pointing positions sampled a range of primary beam offsets extending beyond the GCRT offset as observed on 2003 Sep 28. These observations were sufficiently short such that rotation in feed parallactic angle within any particular pointing position were negligible. For all the off-axis positions after calibration, the maximum value of Stokes $\mathrm{V}$ was found to be 0.02 times Stokes I. Hence, the error in Stokes V within the FWHM of the antennas is $\leq 2 \%$ of Stokes I for an unpolarized source and $<5 \%$ for a high linearly polarized source at $325 \mathrm{MHz}$ band of GMRT.

A variant of multi-resolution Clean was used to better image the non-variable and extended field sources. After amplitude and phase self-calibration of non-variable sources, all sources except one were subtracted from the $u v$-data and the inverse of the amplitude 


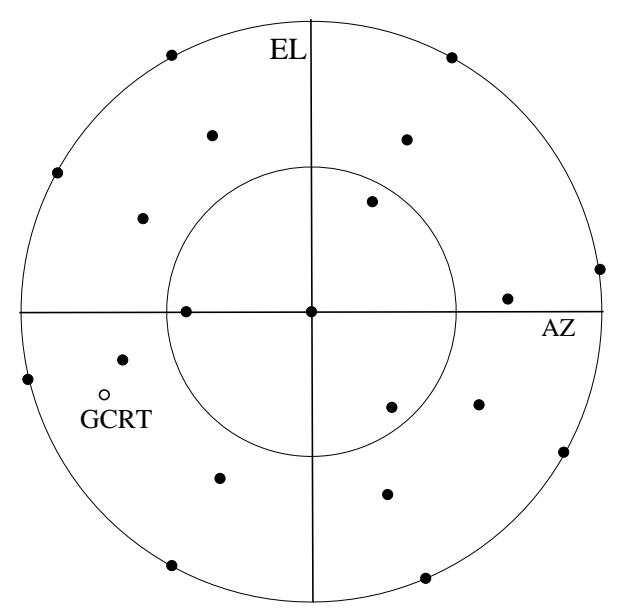

Fig. 1.- 3C48 observed through 20 different parts of the primary beam. The outer circle indicates the FWHM of the primary beam (radius 45') and the inner circle is half of that distance. Azimuth is along X-axis, and elevation along Y-axis. The location of the GCRT is indicated by an open circle.

self-calibration table applied to avoid changes in the flux density of the GCRT during imaging. Subsequently, the GCRT and the strong compact field source (G358.917+0.073, located $0.2^{\circ}$ away from the phase center) not subtracted in the above step were imaged simultaneously from every time sample in the data to confirm the amplitude stability of non-variable sources. The rms noise in the maps are $\sim 34$ mJy.beam ${ }^{-1}$ in each polarization. The equivalent Stokes I noise $\sim 25$ mJy.beam $^{-1}$ is approximately a factor of 2 lower than in Hyman et al. 2006. We attribute the significant reduction in noise due to better removal of extended sources before imaging GCRT coupled with more aggressive inner uv cutoffs (3.3 k-Lambda vs. 2 k-Lambda employed by Hyman et al. 2006). 


\section{Results}

Light curves of the unresolved source, the GCRT, during its active state on September 28, 2003 along with the unrelated compact field source G358.917+0.073 using their peak flux densities are shown in Fig. 2 (top panel) for both the polarizations.

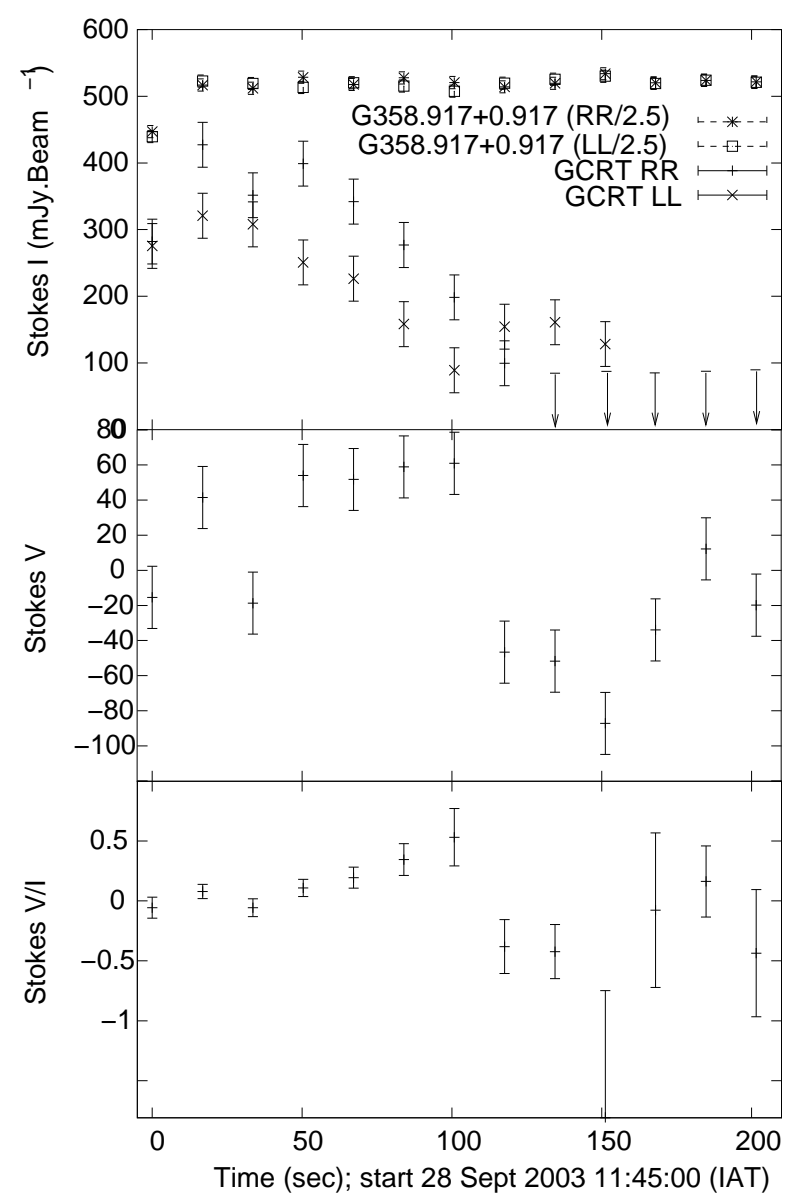

Fig. 2.- Light curves of the GCRT and an unrelated compact field source G358.917+0.073 scaled down by 2.5 for both RR and LL (top). The arrows are $3 \sigma$ upper limits for nondetections. Other 2 panels show Stokes $\mathrm{V}$ at the same timestamps as above (middle) and the ratio of Stokes V to I (bottom).

Due to a correction in data timestamps produced by upgraded observatory software, 
the time used in this paper lags by 7.4 sec relative to Hyman et al. (2006). The peak flux density of both the sources are smaller for the first data point, which is erroneously reduced by the data acquisition software at the start of data acquisition scans. The plot shows that while the flux density for both the polarizations of the unrelated field source are equal and steady within the error-bars, the flux density of the GCRT varies systematically with time. At the start of the scan, the RR flux density is higher than the LL, but it drops almost exponentially to zero after about 200 seconds and after which the GCRT could not be identified. Due to lower rms noise than Hyman et al. (2006), we have been able to detect the source for $\sim 1.5$ times longer duration than reported earlier.

Hyman et al. (2006) searched for circular polarization from this source in a map made using data averaged over the time scale of emission and derived an upper limit of 15\%. However, because the difference between the two polarizations changes sign within the averaging time, their ability to constrain stokes $\mathrm{V}$ was smeared out. Adopting their approach during our re-analysis yields a stokes $\mathrm{V}$ of $12 \%$, consistent with their reported limit. More recent reanalysis of its 2002 outburst (Spreeuw et al. 2009) yields an upper limit of $8 \%$ on the fraction of circular polarization averaged over the emission timescale. To study the variation of the circular polarization, we have also made maps of the GCRT in Stokes V, and a light curve made from these maps is shown in Fig. 2 (middle panel). In Stokes $\mathrm{V}$, the rms noise in the map is $\sim 17 \mathrm{mJy}$ beam $^{-1}$, quite close to the expected thermal noise.

The figure shows that the sign of Stokes V reverses after about 100 sec from the start of the scan and within the data averaging time of $\sim 17$ sec. If the fastest variation in the source properties approach the velocity of light, the emission region would be smaller than $8 \mathrm{R}_{\odot}$. Fig. 2 (bottom panel) shows the ratio of Stokes V to I obtained by dividing the Stokes V maps by the Stokes I maps of the same timestamps using COMB in Aips. It shows the fractional circular polarization is initially low, but increases with time. After 100 seconds 
from the start of the scan it reverses sign. The fraction of circular polarization approaches $\sim 100 \%$ about 150 seconds from the start of the scan. Fig. 3 shows a gray scale map of the difference of Stokes V images made from data integrated between 35 to 103 and 103 to 155 seconds from the start of scan, respectively, during which the sign of stoves $\mathrm{V}$ reversed. This result $(>6 \sigma)$ represents our highest significance detection of Stokes-V from the GCRT.

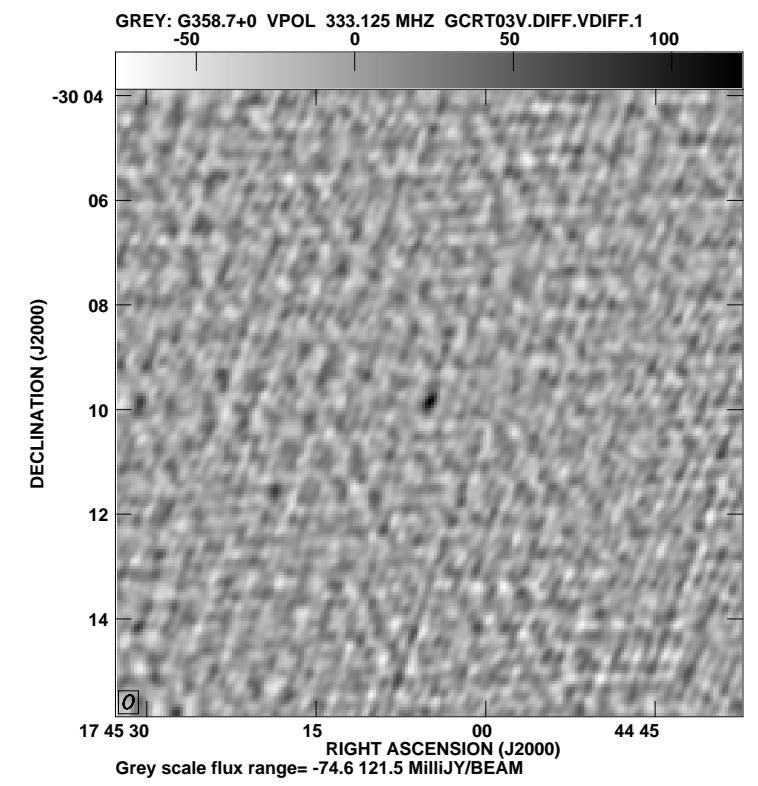

Fig. 3.- Stokes V difference image made from data integrated between 35 to 103 and 103 to 155 seconds respectively from the start of scan and bridging the polarization reversal. Rms noise 19.2 mJy.Beam ${ }^{-1}$. The location of the GCRT is at the center.

Due to large closure errors on the calibrators with lower side band data from the correlator, the bandwidth of the present analysis was limited to $16 \mathrm{MHz}$. The measured Stokes I spectral index $\left(S \propto \nu^{\alpha}\right)$ is $-4 \pm 3$, which is better constrained than in Hyman et al. (2006) $(-4 \pm 5)$. We have also reanalyzed the September 2002 and March 2004 GCRT 
detections. However, we could not detect any significant circular polarization from those 2 epochs, and our upper limits remain as in Hyman et al. (2005) and Hyman et al. (2007) respectively.

\section{Discussions}

\subsection{Emission mechanism}

From the measured flux density and the decay time scale $(\sim 2 \mathrm{~min})$ of the 2002 bursts, Hvman et al. (2005) placed an upper limit of $70 \mathrm{pc}$, which has been revised to 14 pc (Spreeuw et al. 2009) for incoherent emission. Adopting the $\sim 17$ sec time scale for polarization reversal derived from our new analysis of the detected 2003 burst as the fastest variation yields an upper limit of $8 \mathrm{R}_{\odot}$ on its size. Assuming the above size of emission region in 2002 emission lowers the distance limit to $\sim 10$ pc. Given a very low probability of encountering an object within $1 \mathrm{pc}$ and taking this as a distance lower limit still implies a brightness temperature $>10^{10} \mathrm{~K}$. Such a high brightness temperature and circular polarization fraction reaching $\sim 100 \%$ rules out thermal emission and incoherent non-thermal emission like synchrotron and gyro-synchrotron (Melrose \& Dulk 1982), and indicates a coherent emission mechanism. Coherent radiation is known to arise from 3 different processes, (i) pulsar emission, (ii) electron cyclotron maser emission and (iii) plasma emission, which are discussed below.

Pulsar emission could produce highly circularly polarized emission within a single pulse. However, the integration time $(16.8 \mathrm{sec})$ used in the data is longer than the pulse period of any known pulsar, and circular polarization fraction of pulsars goes down when averaged over a full pulse and is typically found to be significantly less than $100 \%$ near an observing frequency of $325 \mathrm{MHz}$ (D. Mitra, private communication). This argues against 
any pulsar-based model as an explanation for the observed properties of the GCRT. Electron cyclotron maser emission occurs at the electron gyro-frequency and its harmonics and could also produce highly circularly polarized emission (Dulk 1985). If the GCRT emission at $330 \mathrm{MHz}$ was due to cyclotron emission at its fundamental frequency, the required magnetic field is $\sim 120$ Gauss and $\sim 2$ lower at the second harmonic. These are typical values of magnetic field in a stellar corona. The relative bandwidth of emission is $\Delta \nu / \nu \approx v_{0}^{2} / c^{2}$ for electrons with speed $v_{0}$, and is typically $\sim 0.01-0.1$ (van den Oord \& de Bruyn 1994). We note, however, that the individual spikes of emission could have durations much smaller than our integration time and could have occured from different physical locations. A significant variation of magnetic fields within these spikes of emission would cause a variation of emitting frequencies causing a broadening of observed bandwidth. This could account for $\gtrsim 30 \mathrm{MHz}$ bandwidth observed in Hyman et al. (2007). The very steep spectral index reported in Hyman et al. (2007) could also be explained if the peak of emission at that time occured at $\lesssim 310 \mathrm{MHz}$.

Plasma emission occurs at the plasma frequency or one of its lower order harmonics. It involves plasma instabilities and subsequent conversion of a fraction of its energy to electromagnetic radiation. Different mechanisms of generating plasma instabilities are known (van den Oord \& de Bruyn 1994). Common characteristics of these are narrow band emission with a high level (approaching 100\%) of circular polarization and high brightness temperature at meter wavelengths. The typical bandwidth of plasma emission is $\Delta \nu / \nu \sim 0.01$. However, as described above, there could be several spots of emission slightly differing in frequency, which would also explain a bandwidth of $>32 \mathrm{MHz}$ as reported in Hyman et al. (2007). We note that plasma frequency of $330 \mathrm{MHz}$ corresponds to an ion density of $\sim 10^{9}$ ions.cm ${ }^{-3}$, which is less likely but cannot be ruled out in the corona of a brown dwarf. However, untill the magnetic field strengths or ion density could be measured 
near the object, any electron cyclotron or plasma emission mechanism attributable to the object cannot as yet be firmly established.

\subsection{Distance to the GCRT and its classification}

If the upper limit to the brightness temperature $\left(T_{B}\right)$ and the linear size $(L)$ of the emission region is known, an upper limit to its distance (D) can be calculated. For electron cyclotron maser emission, it is found that $T_{B}$ could reach $10^{20} \mathrm{~K}$ as in auroral kilometric radiation (Ergun et al. 2000). This upper limit has also been estimated theoretically (Mel'Nik 1994). For plasma emission, $T_{B}$ is expected to be less than $10^{16} \mathrm{~K}$ (Stepanov et al. 1999, 2001). However, for a certain type of plasma emission from double layers, $T_{B}$ could reach $10^{25} \mathrm{~K}$ (Kuijpers 1989). Therefore, we could easily consider the upper limit on its $T_{B}$ to be $10^{20} \mathrm{~K}$. With $\mathrm{L}<8 \mathrm{R}_{\odot}$ (Sect. 4), the upper limit on its distance is $\sim 100 \mathrm{kpc}$, which indicates that the emission does not originate from another galaxy. However, this limit can be much improved by considering known sources of circular polarisation in the Galaxy, which are not ruled out already.

Most of the known low frequency emitting objects are either synchrotron or thermal sources, from which the circular polarization fraction is less than a few percent. However, flare stars in the Galaxy could emit strong high circularly polarized emission. These objects are typically dwarfs stars of class G to M (mostly M). Some of them could be of size $\sim 0.15$ $\mathrm{R}_{\odot}$ (e.g., UV Cet with spectral type M6). As described in Gershberg (2005), more than 50 observations have been carried out in radio bands towards them from the early days of radio astronomy in 1958 to 2001 and cover a frequency range from $20 \mathrm{MHz}$ to several GHz. The flux densities recorded for these flares at cm wavelengths are typically in millijanskys, and the highest recorded was only a fraction of a Jy. However, at lower frequencies, particularly at decameter wavelengths, flux densities of some of the flares recorded are 
100 Jy (Abdul-Aziz et al. 1995) to $\sim 10^{4} \mathrm{Jy}($ Slee 1963). The highest luminosity of any flare star(s) in the metre wave band is $\sim 230 \mathrm{Jy}$ at $136 \mathrm{MHz}$ as recorded by Slee \& Higgins (1969) towards the Orion nebula located $\sim 400$ pc away. If the luminosity of the GCRT in the 2002 outburst is the same, it would be located at a distance of $\sim 4 \mathrm{kpc}$.

Earlier observations of flare stars showed wide variation in the circular polarization fraction with some of the bursts being unpolarized while others having circular polarization fraction reaching up to 100\% (see e.g., Abada-Simon et al. (1994)). At meter wavelengths, Nelson et al. (1979) observed 40 to $60 \%$ circularly polarized emission in 8 cases from flare stars. Lang et al. (1983) reported observations of AD Leo with circular polarization fraction of about $15 \%$, and the emission properties were explained as electron cyclotron emission. Reversal in the sense of circular polarization was also reported from the above flare star (Jackson et al. 1989). As discussed in Dulk (1985) and Melrose (1980), mode coupling could prevent attainment of a high polarization, which could operate for the GCRT when the circular polarization fraction is small and also explain our observed polarization reversal in the data described above.

Measured Rotation period for stars show a decrease in rotation period towards lower mass $\left(0.1 \mathrm{M}_{\odot}\right)$ objects reaching $\sim 0.1$ day (e.g. in Pleaides) (Irwin et al. 2008). A few of these ultracool dwarfs produce flares at radio frequencies (Burgasser \& Putman 2005). As in Hallinan et al. (2007), within one rotation of the object, there could be two pulses of emission. If this is the case during the GCRT 2002 outbursts, its rotation period is $\sim 0.1$ day, consistent with the above finding. Hence, an ultracool dwarf could be progenitor of the GCRT. Kaplan et al. (2008) have considered the object ' $C$ ' as a possible progenitor of the GCRT. However, emission from an ultracool dwarf (L4.5V) do not produce a good fit to the observed multi-band photometry of the object $\mathrm{C}$. We find the goodness of the fit do not change significantly (rms error $~ 0.3$ magnitude) from Kaplan et al. (2008) results when 
the suggested K7V star is replaced by a $\sim 0.1 \mathrm{M}_{\odot}$ star $\sim 0.1$ Gyr old (Baraffe et al. 1998). This could be a young mid to late $\mathrm{M}$ type of star with $\mathrm{A}_{V} \sim 3.5$, indicating it to be at a distance of $\sim 4 \mathrm{kpc}$. Such a distance matches the $10^{20} \mathrm{~K}$ cyclotron brightness temperature limit (radius $\sim 0.15 \mathrm{R}_{\odot}$ ). However, considering the uncertainty $(\sim 0.1$ magnitude) in the IR magnitudes (Kaplan et al. 2008) and in the theoretical model, the quality of the fit is marginal and object ' $\mathrm{C}$ ' is considered only as a candidate progenitor of the GCRT. We, however, note that a hithertho undetected ultracool dwarf located $\gtrsim 200$ pc (Kaplan et al. 2008) could also be the progenitor of the GCRT.

\section{Conclusions}

We have detected time-varying, highly circularly polarised emission from the GCRT J1745-3009 based on a re-analysis of its outburst on 28th September 2003. The percentage of circular polarization approaching $\sim 100 \%$ commensurate with high brightness temperature $\left(>10^{10} \mathrm{~K}\right.$ for a source $>1 \mathrm{pc}$ away) significantly strengthens the original suggestion of a coherent emission scenario (Hyman et al. 2005), but its properties are inconsistent with a pulsar origin. The time scale of change in sign of the circular polarization allows us to estimate an upper limit of $8 \mathrm{R}_{\odot}$ on its size. Based on its measured emission characteristics, either electron cyclotron maser or plasma emission processes could be the most plausible origin scenarios.

Ascribing the 77 minute periodicity observed in its 2002 outbursts to half the rotation period of its progenitor, observed radio emission of the GCRT could be explained as outbursts from a highly subsolar flare star. Its distance is estimated to be $\leq 4 \mathrm{kpc}$. Independent verification, would however, be required to confirm its progenitor. 


\section{Acknowledgments}

We thank the staff of the GMRT that made these observations possible. GMRT is run by the National Center for Radio Astrophysics of the Tata Institute of Fundamental Research. S.D.H. is supported by funding from Research Corporation. Basic research in radio astronomy at the Naval Research Laboratory is supported by 6.1 base funding. 


\section{REFERENCES}

Abada-Simon, M., Lecacheux, A., Louarn, P., et al. 1994, A\&A, 288, 219

Abdul-Aziz, H., Abranin, E. P., Alekseev, I. Y., et al. 1995, A\&AS, 114, 509

Baraffe, I., Chabrier, G., Allard, F., \& Hauschildt, P. H. 1998, A\&A, 337, 403

Burgasser, A. J. \& Putman, M. E. 2005, ApJ, 626, 486

Dulk, G. A. 1985, ARA\&A, 23, 169

Ergun, R. E., Carlson, C. W., McFadden, J. P., et al. 2000, ApJ, 538, 456

Gershberg, R. E. 2005, Solar-Type Activity in Main-Sequence Stars, ed. R. E. Gershberg

Hallinan, G., Bourke, S., Lane, C., et al. 2007, ApJ, 663, L25

Hyman, S. D., Lazio, T. J. W., Kassim, N. E., et al. 2005, Nature, 434, 50

Hyman, S. D., Lazio, T. J. W., Roy, S., et al. 2006, ApJ, 639, 348

Hyman, S. D., Roy, S., Pal, S., et al. 2007, ApJ, 660, L121

Irwin, J., Hodgkin, S., Aigrain, S., et al. 2008, MNRAS, 383, 1588

Jackson, P. D., Kundu, M. R., \& White, S. M. 1989, A\&A, 210, 284

James, D. J., Jardine, M. M., Jeffries, R. D., et al. 2000, MNRAS, 318, 1217

Jeffries, R. D., Burleigh, M. R., \& Robb, R. M. 1996, A\&A, 305, L45+

Kaplan, D. L., Hyman, S. D., Roy, S., et al. 2008, ApJ, 687, 262

Kuijpers, J. 1989, Sol. Phys., 121, 163

Kulkarni, S. R. \& Phinney, E. S. 2005, Nature, 434, 28 
Kundu, M. R., Jackson, P. D., White, S. M., \& Melozzi, M. 1987, ApJ, 312, 822

Lang, K. R., Bookbinder, J., Golub, L., \& Davis, M. M. 1983, ApJ, 272, L15

Mel'Nik, V. N. 1994, Kinematika i Fizika Nebesnykh Tel, 10, 77

Melrose, D. B. 1980, Plasma astrohysics. Nonthermal processes in diffuse magnetized plasmas - Vol.1: The emission, absorption and transfer of waves in plasmas; Vol.2: Astrophysical applications (New York: Gordon and Breach, 1980)

Melrose, D. B. \& Dulk, G. A. 1982, ApJ, 259, 844

Nelson, G. J., Robinson, R. D., Slee, O. B., et al. 1979, MNRAS, 187, 405

Slee, O. B. 1963, Nature, 199, 991

Slee, O. B. \& Higgins, C. S. 1969, Nature, 224, 1087

Spreeuw, H., Scheers, B., Braun, R., et al. 2009, A\&A, 502, 549

Stepanov, A. V., Kliem, B., Krüger, A., Hildebrandt, J., \& Garaimov, V. I. 1999, ApJ, 524, 961

Stepanov, A. V., Kliem, B., Zaitsev, V. V., et al. 2001, A\&A, 374, 1072

Turolla, R., Possenti, A., \& Treves, A. 2005, ApJ, 628, L49

van den Oord, G. H. J. \& de Bruyn, A. G. 1994, A\&A, 286, 181

Zhang, B. \& Gil, J. 2005, ApJ, 631, L143

Zhu, W. W. \& Xu, R. X. 2006, MNRAS, 365, L16 